\title{
M. WIDMER
}

\section{Évaluation des performances d'un atelier flexible en tenant compte des pannes et des maintenances}

Revue française d'automatique, d'informatique et de recherche opérationnelle. Recherche opérationnelle, tome 21, n 2 (1987), p. 137-151.

<http://www.numdam.org/item?id=RO_1987_21_2_137_0>

(C) AFCET, 1987, tous droits réservés.

L'accès aux archives de la revue « Revue française d'automatique, d'informatique et de recherche opérationnelle. Recherche opérationnelle » implique l'accord avec les conditions générales d'utilisation (http://www.numdam.org/ legal.php). Toute utilisation commerciale ou impression systématique est constitutive d'une infraction pénale. Toute copie ou impression de ce fichier doit contenir la présente mention de copyright.

\section{Numdam}

Article numérisé dans le cadre du programme

Numérisation de documents anciens mathématiques

http://www.numdam.org/ 


\title{
ÉVALUATION \\ DES PERFORMANCES D'UN ATELIER FLEXIBLE EN TENANT COMPTE DES PANNES ET DES MAINTENANCES $\left({ }^{*}\right)$
}

\author{
par M. WIDMER $\left(^{1}\right)$
}

\begin{abstract}
Résumé. - Parmi les méthodes mathématiques connues pour estimer les performances des ateliers flexibles, peu d'entre elles tiennent compte des perturbations troublant le bon fonctionnement de latelier. Ce papier présente une manière simple de modéliser ces perturbations, puis décrit les transformations à apporter aux formules d'évaluation des performances basées sur la théorie des réseaux de files d'attente. Une comparaison entre des résultats obtenus par l'analyse d'une part et par la simulation d'autre part est exposée à la fin du texte.
\end{abstract}

Mots clés : Perturbations; ateliers flexibles; réseaux de files d'attente.

Abstract. - Few of known mathematical methods to compute the Flexible Manufacturing Systems performances take in account perturbations disturbing the smooth working of the factory. This paper describes a simple modelling for these perturbations and presents how to transform the performance evaluation formulas based on the queuing network theory. The last section compares the analytical results with simulation performances.

Keywords : Perturbations; Flexible Manufacturing Systems; queuing networks.

\section{INTRODUCTION}

Dans les systèmes de production actuels, l'automatisation prend une place de plus en plus importante. Après l'introduction des machines à commande numérique, les ateliers mécaniques se transforment progressivement en ateliers flexibles. Une définition relativement précise de ces derniers est proposée dans Pru [84]:

«Un atelier flexible permet la production automatique de pièces de types divers et en quantités variables. Les opérateurs n'interviennent pas directement dans le processus de fabrication et limitent essentiellement leurs interventions

(*) Reçu août 1986.

(1) École Polytechnique Fédérale de Lausanne, Département de Mathématiques, Chaire de Recherche Opérationnelle, CH-1015 Lausanne.

R.A.I.R.O. Recherche opérationnelle/Operations Research, 0399-0559/87/02 137 15/\$3.50

(C) AFCET-Gauthier-Villars 
à l'entretien; l'ordonnancement de la production est géré par un système informatique." "

L'évaluation des performances des ateliers flexibles peut se faire en développant des logiciels de simulation très sophistiqués tenant compte, par exemple, de la nature et du nombre de machines, des gammes opératoires, du réseau et des moyens de manutention, de l'emplacement et de la capacité des aires de stockage. La création de tels logiciels demande un investissement non négligeable si toutes les contraintes sont explicitement prises en considération.

Une méthode rapide pour évaluer ces performances nous est fournie par la théorie des réseaux de files d'attente $(\operatorname{Cav}[])$. Au stade d'un avantprojet, lorsqu'il faut déterminer le nombre de composantes pour concevoir un système performant, on ne considère que les machines et les gammes opératoires. L'atelier est présenté comme un ensemble de stations avec des files d'attente de capacité illimitée entre lesquelles circulent différents clients (les tâches). Cette structure est analogue aux réseaux de files d'attente. Un logiciel, développé par Solberg (Sol[80]), utilise cette méthode pour tester l'efficacité des ateliers et est déjà largement diffusé aux États-Unis.

Si on étudie la littérature consacrée à l'application de diverses théories mathématiques aux ateliers flexibles, on constate que trop peu d'articles traitent les perturbations momentanées pouvant entraver le bon fonctionnement de l'atelier (à l'état d'équilibre): les pannes et les maintenances préventives. Deux approches ont été envisagées.

La première, due à L. Fortuin (For [84]), a pour hypothèse de base que la durée entre deux pannes suit une distribution de Weibull. Fortuin propose un algorithme pour estimer les performances et met aussi en lumière le principal inconvénient de cette méthode: l'estimation des paramètres de la loi de Weibull. De plus, il n'associe pas les pannes aux machines mais à l'atelier tout entier.

La seconde approche est exposée par Buzacott et Shanthikumar (Buza [80]). La mesure la plus simple pour comparer les performances de divers systèmes étant le taux de production, ils introduisent l'effet des pannes en tenant compte des éléments suivants: le taux de pannes d'une machine par cycle, le temps de réparation et la capacité de stockage. Ils proposent une formule permettant de calculer le rapport entre le taux de production avec pannes et le taux de production sans panne. Malheureusement, celle-ci ne s'applique que lorsque toutes les machines sont identiques.

Le but de ce papier est de proposer une nouvelle approche des perturbations pour évaluer les performances d'un atelier. 


\section{PRESENTATION DE LA NOUVELLE APPROCHE}

L'idée de base est la suivante: les pannes et les maintenances sont directement liées aux machines. En effet, chaque machine a sa propre fiabilité et des besoins en maintenance particuliers. De plus, lorsqu'une machine est " perturbée ", elle est occupée mais elle ne produit rien. Le fait de créer deux produits fictifs (un par type de perturbations) est donc justifié: un produit fictif occupe une machine pendant une certaine période (la durée de la perturbation). La valeur économique d'un produit fictif étant nulle par définition, ceci revient à dire que la machine ne fabrique rien durant cette période. De plus, la durée d'occupation de la machine par la pièce fictive étant différente d'une machine à l'autre, chaque machine a ses propres caractéristiques de pannes et de maintenance.

C'est en partant de cette idée simple pour modéliser les pannes et les maintenances que certains résultats de la théorie des réseaux de files d'attente ont été adaptés pour tenir compte des perturbations. Une modélisation complète est présentée dans le paragraphe suivant.

\section{NOTATIONS, HYPOTHÈSES ET MODÉLISATION}

Avant de proposer une modélisation du système, il convient de bien définir quelques notions de production.

L'atelier flexible est capable de fabriquer divers types de pièces simultanément. Chaque produit $r$ représente une fraction $f c_{r}$ de la production globale $\left(\sum_{r} f c_{r}=1\right)$. Le traitement d'un produit se compose d'une suite d'opérations, une opération étant définie comme l'usinage (ou l'assemblage) d'une pièce sur une machine. A chaque opération est associé un temps d'exécution.

Toutes les opérations ne doivent pas être faites systématiquement pour chaque pièce d'un produit donné. Par exemple, l'inspection de la qualité des pièces peut se faire par le contrôle d'une pièce sur dix ou d'une pièce sur cent. On détermine ainsi la fréquence d'une opération sur une machine.

Chaque produit a une certaine valeur. Il peut s'agir du bénéfice que rapporte le produit ou, si la fabrication du produit se fait par fournées (batch), la valeur correspond au nombre de pièces se trouvant sur le montage de la palette.

Pour la modélisation du système, les hypothèses générales présentées par Buzacott et Shanthikumar (Buza [83]) sont reprises partiellement. 
(i) L'atelier est représenté comme un système fermé dans lequel circulent $N$ palettes; celles-ci sont susceptibles de recevoir n'importe quel type de pièces.

(ii) L'atelier est composé de $M$ machines. Les machines peuvent avoir un ou plusieurs serveurs, chaque serveur ne pouvant exécuter plus d'une tâche simultanément. Ces $M$ machines sont desservies par un système de transport.

(iii) La discipline d'attente est FIFO (premier arrivé, premier servi).

(iv) On suppose qu'il n'y a pas de problème de stockage (il y a une infinité de places de stockage).

(v) On considère $p \geqq 1$ classes de produits (i. e. $p$ produits différents); de plus, on ajoute les deux produits fictifs ( $p$ p et $\| m »)$; pour les calculs, $R=p+2$ produits sont pris en compte. On introduit :

$q_{i r}$ est la probabilité qu'un produit de la classe $r$ ait sa première opération sur la machine $i$.

(vi) Pour chaque classe $r$, le cheminement du produit est déterminé par une matrice de probabilité $P_{i j, r}=$ probabilité d'aller de la machine $i$ à la machine $j$.

$1-\sum_{j} P_{i j, r}$ donne la probabilité de quitter le système après avoir été traité par la machine $i$.

(vii) Le nombre moyen de visites d'un produit $r$ sur la machine $i$ vaut

$$
e_{i r}=q_{i r}+\sum_{j} P_{j i, r} * e_{j r} \quad(i=1 \ldots M)
$$

(viii) Le temps d'exécution d'un produit $r$ sur la machine $i$ est une variable aléatoire exponentielle de moyenne $t_{i r}=1 / \mu_{i r}$; il est à noter que ce temps est lié à la machine $e t$ au produit.

Le temps d'exécution moyen de la machine peut être calculé de la manière suivante:

$$
t_{i}=\frac{\sum_{r} t_{i r} * f c_{r}}{\sum_{r} e_{i r} * f c_{r}}=\frac{1}{\mu_{i}} .
$$

Il convient de préciser certaines notions définies ci-dessus pour les produits particuliers $" p$ " et $" m$ ». Comme tout ce qui suit est valable pour les produits " $p$ » (correspondant aux pannes) et $" m$ " (pour les maintenances), seules des explications seront fournies pour " $p$ ".

La fraction de la production totale dédiée à " $p$ " $\left(f c_{p}\right)$ est le taux de pannes du système; la valeur de " $p$ » est nulle. 
Le produit " $p$ » peut subir $m$ opérations (une par machine). La fréquence d'une opération sur la machine $i$ s'interprète comme le taux de pannes perturbant la machine $i$. Le temps d'exécution d'une panne sur la machine $i$ est la durée moyenne d'une panne sur la machine $i$.

L'élément $q_{i p}$ représente la probabilité qu'une panne affecte la machine $i$ et la matrice de probabilité $P_{i j, p}$ est nulle (après avoir bloqué une machine, une panne « quitte » l'atelier).

\section{LES MODIFICATIONS A APPORTER AUX FORMULES}

Un élément important pour ce qui va suivre est la fraction de production dédiée aux produits réels.

$$
F=\sum_{j \neq p, m} f c_{j}=1-f c_{p}-f c_{m}
$$

Dans le cas sans perturbation, la proportion de chaque produit est connue. Lorsque les pannes et les maintenances interviennent, il faut ajuster ces fractions de production (notons $\tilde{f} c_{i}$ la proportion sans perturbation):

$$
f c_{i}:=\tilde{f} c_{i} * F \text {. }
$$

La théorie basée sur les réseaux de files d'attente nous permet de calculer les éléments suivants: (Cav[ ], Dub [83], Sch [77], Sol [80]):

$a$. Les taux de production.

b. Les temps moyens: passé dans le système, en exécution, en transfert, en attente.

c. La machine " bouchon ».

$d$. Pour chaque machine: l'utilisation, le nombre moyen d'articles dans la machine, en exécution, en attente.

Reprenons un à un les divers points énoncés ci-dessus et exhibons les modifications à apporter pour avoir une estimation des performances réelles de l'atelier en tenant compte des perturbations.

\section{4a. Les taux de production}

Pour un système composé de $M$ machines et $N$ palettes, le taux de production (throughput) est donné par (Dub [83]) : pour $N>1$.

$$
T H(N)=\lambda * G(M, N-1) / G(M, N)
$$


où $\lambda$ est le taux d'arrivées dans le système, $G(M, N)$ est la constante de normalisation des réseaux fermés de Jackson.

L'inconvénient, pour un cas réaliste, c'est qu'il existe plusieurs produits "fabricables» dans un même atelier et que le $\lambda$ est un taux global. Le taux de production $T H(N)$ est ainsi un taux de production global, confondant les divers produits pour en faire un produit global.

Solberg (Sol [80]) propose un $\lambda$ qui tienne compte de la diversité des produits. En connaissant la fraction de la production totale attribuée à chaque produit $r\left(f c_{r}\right)$ et le nombre moyen de visites du produit $r$ à la machine $i\left(e_{i r}\right)$, on peut calculer le nombre moyen d'opérations pour finir un produit "global ":

$$
O M=\sum_{r} f c_{r} * \sum_{i} e_{i r}
$$

Comme deux opérations différentes s'effectuent sur deux machines différentes, la fréquence des visites à la station de transport est

$$
f_{T}=1 / O M \text {. }
$$

La fréquence des visites à la station de transport peut être assimilée au taux d'arrivées dans le système.

Ainsi, le taux de production de la formule (3) se transforme en :

$$
T H(N)=f_{T} * G(M, N-1) / G(M, N) .
$$

Ceci donne le taux de production fictif (avec " $p$ " et " $m$ " comme produits). Le taux de production réel est

$$
T H^{\prime \prime}(N)=F * T H(N) \text {. }
$$

La fréquence réelle des visites à la station de transport est redéfinie:

$$
f_{T}^{\prime \prime}=F * f_{T}
$$

Ceci représente l'occupation du transporteur avec des produits réels.

Pour le cas particulier $N=1$, le taux de production est donné par:

$$
T H(1)=f_{T} / G(M, 1) \text {. }
$$

Si on ne considère que les produits réels:

$$
T H^{\prime \prime}(1)=f_{T}^{\prime \prime} / G(M, 1)=F * T H(1) .
$$


Il existe une différence essentielle entre ce qui est présenté ici et ce que proposent Buzacott et Shanthikumar (Buza [80]). Ils calculent un rapport entre la production effective avec pannes et la production effective sans panne, alors qu'ici $T H^{\prime \prime}(N)$ représente la production effective en produits réels avec pannes et $T H(N)$ représente la production fictive (produits réels et produits fictifs) avec pannes.

4 b. Les temps moyens: passé dans le système, en exécution, en transfert, en attente

Le temps moyen passé dans le système est donné par la formule de Little (pour $N \geqq 1$ )

$$
T(N)=N / T H(N)
$$

qui devient dans notre cas

$$
T^{\prime \prime}(N)=N / T H^{\prime \prime}(N)=T(N) / F
$$

On a bien sûr: $T^{\prime \prime}(N) \geqq T(N)$; ceci est raisonnable: les pannes et les maintenances perturbant le système, le temps moyen passé dans l'atelier se trouve ainsi rallongé.

Le temps moyen de convoyance est fourni par

$$
T_{c}=t_{T} / f_{T}
$$

où $t_{T}$ est le temps moyen de transport calculé sur tous les transports possibles dans l'atelier.

Dans notre cas, l'ajustement est le suivant:

$$
T_{c}^{\prime \prime}=t_{T} / f_{T}^{\prime \prime}=T_{c} / F
$$

Le temps moyen d'exécution s'exprime comme suit:

$$
T_{e}=\left(G(M, 1)-t_{T}\right) / f_{T}=G(M, 1) / f_{T}-T_{c}
$$

où $G(M, 1) / f_{T}$ est le temps de production d'un produit dans le cas où une seule palette circule dans l'atelier.

Dans notre cas,

$$
T_{e}^{\prime \prime}=\left(G(M, 1)-t_{T}\right) / f_{T}^{\prime \prime}=T_{e} / F
$$

Finalement, le temps moyen d'attente n'est autre que:

$$
T_{a}=T(N)-T_{c}-T_{e}
$$


et ajusté dans notre cas

$$
T_{a}^{\prime \prime}=T^{\prime \prime}(N)-T_{c}^{\prime \prime}-T_{e}^{\prime \prime}=T_{a} / F
$$

\section{4 c. La machine «bouchon»}

Pour $N=\infty$, le temps moyen passé dans le système est naturellement infini. Le taux de production est défini par le rapport:

$$
T H(\infty)=f_{T} / \max _{i}\left(w_{i} / s_{i}\right)
$$

où $w_{i}$ est la charge fictive de la machine $i$ (en tenant compte de " $p$ » et « $m$ » comme produits), $s_{i}$ est le nombre de serveurs de la machine $i$.

Dans notre cas

$$
T H^{\prime \prime}(\infty)=f_{T}^{\prime \prime} / \max _{i}\left(w_{i} / s_{i}\right)=F * T H(\infty)
$$

Pour déterminer la machine qui créera un engorgement si le nombre de palettes augmente (station "bouchon»), il faut travailler avec la charge fictive car les perturbations contribuent à charger artificiellement les machines. Une machine peu fiable peut devenir ainsi une station " bouchon " même si elle est très peu chargée par les produits réels.

4 d. Pour chaque machine: l'utilisation, le nombre moyen de produits dans la machine, en exécution, en attente

Pour le calcul de $G(M, N)$, la constante de normalisation de Jackson, il faut évaluer la charge de la machine. $G(M, N)$ a été calculée avec la charge fictive $w_{j}$ (due aux produits réels et aux produits fictifs).

$$
w_{j}=f_{j} * t_{j}
$$

avec $t_{j}$ le temps moyen d'exécution de la machine $j, f_{j}$ la fréquence des visites à la machine $j$

$$
f_{j}=\frac{\sum_{r} e_{j r} * f c_{r}}{\sum_{r} f c_{r} * \sum_{j} e_{j r}}
$$


La fréquence des visites de produits réels à la machine $j$, est

$$
f_{j}^{\prime \prime}=f_{j} \frac{e_{j m} * f c_{m}+e_{j p} * f c_{p}}{\sum_{r} f c_{r} * \sum_{j} e_{j r}}
$$

La charge réelle de la machine $j$ se déduit de là :

$$
w_{j}^{\prime \prime}=f_{j}^{\prime \prime} * t_{j}^{\prime \prime}
$$

avec $t_{j}^{\prime \prime}$ le temps moyen d'exécution de la machine $j$ en ne tenant compte que des produits réels.

Finalement, les utilisations fictive et réelle sont données par:

$$
\begin{array}{ll}
\text { fictive: } & u_{j}=\left(w_{j} / s_{j}\right) * G(M, N-1) / G(M, N) \\
\text { réelle: } & u_{j}^{\prime \prime}=\left(w_{j}^{\prime \prime} / s_{j}\right) * G(M, N-1) / G(M, N)
\end{array}
$$

Le nombre moyen de produits dans la machine $j$ est fourni par (Dub [83]):

$$
L_{j}(N)=\sum_{i} i * p_{i j}(N)
$$

où $p_{i j}(N)$ est la probabilité marginale d'avoir $i$ palettes dans la machine $j$ lorsqu'on a $N$ palettes qui circulent dans le système.

Or, $p_{i j}(N)$ est une fonction de la charge $w_{j}$ et ainsi $L_{j}(N)$ est une fonction de la charge $w_{j}$. On peut donc estimer le nombre moyen d'articles réels dans la machine en connaissant la charge réelle $w_{j}^{\prime \prime}$.

$$
L_{j}^{\prime \prime}(N)=\text { fonction }\left(w_{j}^{\prime \prime}\right)=\sum_{i} i * p_{i j}^{\prime \prime}(N)
$$

De là, on déduit aisément le nombre moyen d'articles en exécution

$$
A E_{j}^{\prime \prime}=u_{j}^{\prime \prime} * s_{j}
$$

et le nombre moyen d'articles en attente

$$
A A_{j}^{\prime \prime}=L_{j}^{\prime \prime}(N)-A E_{j}^{\prime \prime} .
$$

Il est bien sûr possible de calculer d'autres éléments, comme la répartition des temps par opérations, par articles, etc. Les exprimer ici rendrait cette énumération de formules encore plus fastidieuse. 


\section{COMPARAISON ENTRE LA THÉORIE ET LA PRATIQUE}

Afin d'illustrer ce qui précède, un exemple est présenté dans ce paragraphe. Les milieux industriels étant peu enclins à fournir des données (surtout au sujet des pannes), les données utilisées sont fictives.

L'atelier pris en considération est composé de 6 machines et fabrique 4 types de pièces différents. Les produits cheminent dans l'atelier suivant le principe du "flow-shop ». Les temps d'exécution des pièces sur les diverses machines sont fournis par le tableau I.

Les tableaux II et III donnent la durée moyenne des pannes et des maintenances sur chaque machine, ainsi que la probabilité que, lorsqu'une perturbation se manifeste dans l'atelier, elle ait lieu sur une machine donnée.

Finalement, les temps de transport entre les diverses machines sont présentés dans le tableau IV.

Le logiciel utilisé pour simuler le comportement de l'atelier a été écrit en Turbo-Pascal et développé sur un PC.

Toutes les durées (exécution, pannes, maintenances) sont des variables aléatoires distribuées suivant une loi exponentielle. Les arrivées dans le système suivent une loi de Poisson. Le temps entre deux pannes sur une même machine suit une loi de Weibull et celui entre deux maintenances suit une loi exponentielle.

Soixante simulations de 10000 unités de temps ont été effectuées. Vingt d'entre elles tiennent compte des deux types de perturbations, les autres ne prenant en considération qu'un seul type de perturbations, et ceci afin de pouvoir déterminer les taux de pannes et de maintenances.

Le tableau $\mathrm{V}$ présente une synthèse des résultats obtenus: il présente le taux de production et l'utilisation des machines en fonction du nombre de palettes. Les performances de l'atelier idéal, c'est-à-dire du système où les machines ne sont pas affectées par les perturbations, peuvent être lues dans la ligne $E$.

Pour le modèle analytique, l'utilisation des machines par les produits réels (ligne A 1) est calculée par la formule 26 et l'occupation des machines par les produits et les perturbations (ligne A 2) est évaluée grâce à la formule 25 . Ainsi, pour déterminer la véritable influence des pannes et des maintenances sur le système, il faut comparer la ligne $E$ avec celles d'indice 1 . 
Il est intéressant de constater que les machines 4 et 6 ont la même utilisation théorique (cas sans perturbation). Cependant, la machine 4 étant moins fiable, son utilisation par les produits réels est inférieure de $2 \%$ par rapport à celle de la machine 6.

Malgré de légères différences dans les hypothèses de base (temps de transport constant et intervalle entre deux pannes suivant une loi exponentielle pour le modèle analytique contre temps de transport variable et intervalle entre deux pannes suivant une loi de Weibull pour la simulation), les résultats confirment le bienfondé de la théorie. En effet, l'erreur la plus grande sur l'estimation du taux de production est de $6,6 \%$ (pour $N=10$ palettes). De plus, le taux d'utilisation réelle des machines est approché avec moins de $3 \%$ d'erreur dans quatre cas sur cinq.

Ainsi, cette nouvelle méthode d'évaluation permet de traiter les perturbations de manière très efficace.

\section{CONCLUSION}

Par cette modélisation des pannes et des maintenances, il est possible d'introduire les perturbations dans l'évaluation des performances des ateliers de productions. Cependant, il faut relever un problème de données: si les gammes opératoires des produits réels sont faciles à connaître (temps d'exécution), l'estimation des temps moyens et des fréquences des arrêts est plus délicate. Mais comme la plupart des équipes de maintenance des ateliers de production tiennent à jour un cahier des réparations effectuées sur les diverses machines, il suffit de traiter cette information pour obtenir les éléments nécessaires aux calculs. De plus, on constate que pour " ajuster " les taux de production et les temps moyens, il est possible de modifier des logiciels existants comme CAN-Q (Sol [80]) en introduisant les pannes et les maintenances comme des produits et en évaluant le coefficient $F$.

La méthode d'évaluation présentée ici est rapide et peut être facilement implantée sur n'importe quel micro-ordinateur. Il faut être conscient du fait que les résultats fournis par la théorie des réseaux de files d'attente sont relativement grossiers. Pour des estimations plus fines, la simulation détaillée reste un outil mieux adapté. 


\section{BIBLIOGRAPHIE}

Buza [80] J. A. Buzacott et J. G. Shanthikumar, Models for Understanding Flexible Manufacturing Systems, A.I.I.E. Transactions, december 1980.

Cav [ ] J. B. CAvalllé et D. Dubois, Intérêt de la théorie des réseaux de files d'attente pour l'évaluation des performances d'un atelier flexible, C.E.R.T.-D.E.R.A. Toulouse.

Dub [83] D. DuboIs, A Mathematical Model of a Flexible Manufacturing System with Limited In-Process Inventory, European Journal of Operational Research, ${ }^{\circ}$ 14, 1983.

For [84] L. FortuIn, Expected Demand for Service Parts when Failure Times Have a Weibull Distribution, European Journal of Operational Research, $\mathrm{n}^{\circ} 17,1984$.

Pru [84] F. PRUvot, Introduction aux automates industriels, E.P.F., Lausanne, janvier 1984.

Sch [77] P. J. SchweItZer, Maximum Throughput in Finite-Capacity Open Queuing Network with Production-Form Solutions, Management Science, vol. 24, $\mathrm{n}^{\circ}$ 2, octobre 1977.

Sol [80] J, J. Solberg, $C A N-Q$ User's Guide, School of Industrial Engineering, Purdue University, West Lafayette, Indiana, 1980. 
LES DONNEEES UTILISÉES.

\begin{tabular}{|c|c|c|c|c|c|c|c|}
\hline & \multicolumn{5}{|c|}{ machine } & \\
\hline & & 1 & 2 & 3 & 4 & 5 & 6 \\
\hline \multirow{4}{*}{ piece } & .1 & 2 & 0 & 4 & 2 & 0.5 & 1 \\
\hline & 2 & 4 & 0 & 0 & 3 & 3 & 1.5 \\
\hline & 3 & 1 & 1.2 & 0 & 0 & 2 & 1.5 \\
\hline & 4 & 1.5 & 2 & 6.5 & 0 & 0.5 & 1 \\
\hline
\end{tabular}

TABLEAU II

machine

\begin{tabular}{c|cccccc} 
& 1 & 2 & 3 & 4 & 5 & 6 \\
\hline durée & 15 & 20 & 7.5 & 20 & 15 & 30 \\
prob. & 0.190 & 0.115 & 0.290 & 0.144 & 0.191 & 0.070
\end{tabular}

taux de pannes du système : 0.0830

TABLEAU III

machine

\begin{tabular}{c|cccccc} 
& 1 & 2 & 3 & 4 & 5 & 6 \\
\hline durée & 1 & 2 & 0.5 & 1.5 & 2 & 1 \\
prob. & 0.199 & 0.126 & 0.217 & 0.125 & 0.125 & 0.208
\end{tabular}

taux de maintenances du système : 0.0342 
TABLEAU IV

\section{machine}

\begin{tabular}{c|ccccccc} 
& 1 & 2 & 3 & 4 & 5 & 6 & sortie \\
\hline \multirow{2}{*}{ entrée } & 0.1 & 0.2 & 0.3 & 0.4 & 0.5 & 0.6 & 0.7 \\
1 & 0.0 & 0.1 & 0.2 & 0.3 & 0.4 & 0.5 & 0.6 \\
2 & 0.1 & 0.0 & 0.1 & 0.2 & 0.3 & 0.4 & 0.5 \\
3 & 0.2 & 0.1 & 0.0 & 0.1 & 0.2 & 0.3 & 0.4 \\
4 & 0.3 & 0.2 & 0.1 & 0.0 & 0.1 & 0.2 & 0.3 \\
5 & 0.4 & 0.4 & 0.2 & 0.1 & 0.0 & 0.1 & 0.2 \\
6 & 0.5 & 0.4 & 0.3 & 0.2 & 0.1 & 0.0 & 0.1
\end{tabular}

\section{LES RÉSULTATS.}

Notations pour le type:

$S$ : résultats de la simulation;

$A$ : résultats du modèle analytique;

$E$ : taux de production et utilisation des machines s'il n'y a pas de perturbation;

1: utilisation des machines par les produits réels uniquement;

2: occupation des machınes par les produits et les pertrubations.

\section{TABLeAU V}

\section{$N=10$ palettes}

$\begin{array}{cccccccc}\text { type } & \text { TH(10) } & \text { U1 } & \text { U2 } & \text { U3 } & \text { U4 } & \text { U5 } & \text { U6 } \\ \text { S1 } & 0.2941 & 0.632 & 0.239 & 0.780 & 0.357 & 0.441 & 0.368 \\ \text { S2 } & & 0.713 & 0.320 & 0.835 & 0.432 & 0.520 & 0.435 \\ \text { A1 } & 0.3135 & 0.650 & 0.243 & 0.768 & 0.378 & 0.460 & 0.386 \\ \text { A2 } & & 0.753 & 0.322 & 0.888 & 0.479 & 0.558 & 0.457 \\ \text { E } & 0.3475 & 0.739 & 0.278 & 0.912 & 0.434 & 0.521 & 0.434\end{array}$


$N=15$ palettes

$\begin{array}{lccccccc}\text { type } & \text { TH(15) } & \text { U1 } & \text { U2 } & \text { U3 } & \text { U4 } & \text { U5 } & \text { U6 } \\ \text { S1 } & 0.3239 & 0.692 & 0.258 & 0.851 & 0.412 & 0.495 & 0.403 \\ \text { S2 } & & 0.769 & 0.335 & 0.907 & 0.489 & 0.590 & 0.467 \\ \text { A1 } & 0.3376 & 0.700 & 0.262 & 0.827 & 0.407 & 0.495 & 0.416 \\ \text { A2 } & & 0.811 & 0.346 & 0.957 & 0.516 & 0.601 & 0.492 \\ \text { E } & 0.3700 & 0.786 & 0.296 & 0.971 & 0.463 & 0.555 & 0.463\end{array}$

$N=20$ palettes

$\begin{array}{lllllll}\text { type } T H(20) & \text { U1 } & \text { U2 } & \text { U3 } & \text { U4 } & \text { U5 } & \text { U6 }\end{array}$

$\begin{array}{llllllll}\text { S1 } & 0.3377 & 0.720 & 0.270 & 0.886 & 0.424 & 0.503 & 0.425 \\ \text { S2 } & & 0.804 & 0.338 & 0.944 & 0.506 & 0.591 & 0.484 \\ \text { A1 } & 0.3467 & 0.718 & 0.269 & 0.850 & 0.418 & 0.508 & 0.427 \\ \text { A2 } & & 0.832 & 0.356 & 0.982 & 0.530 & 0.617 & 0.505 \\ \text { E } & 0.3773 & 0.802 & 0.302 & 0.990 & 0.472 & 0.566 & 0.472\end{array}$

$N=25$ palettes

$\begin{array}{llllllll}\text { type } & T H(25) & \text { U1 } & \text { U2 } & \text { U3 } & \text { U4 } & \text { U5 } & \text { U6 }\end{array}$

$\begin{array}{llllllll}\text { S1 } & 0.3472 & 0.725 & 0.280 & 0.927 & 0.427 & 0.527 & 0.436 \\ \text { S2 } & & 0.867 & 0.433 & 0.982 & 0.499 & 0.613 & 0.501 \\ \text { A1 } & 0.3503 & 0.726 & 0.272 & 0.858 & 0.422 & 0.514 & 0.432 \\ \text { A2 } & & 0.841 & 0.359 & 0.993 & 0.535 & 0.623 & 0.510 \\ \text { E } & 0.3797 & 0.807 & 0.304 & 0.997 & 0.475 & 0.570 & 0.475\end{array}$

